\title{
Histone H2B ubiquitination: the cancer connection
}

\author{
Joaquín M. Espinosa ${ }^{1}$ \\ Department of Molecular, Cellular, and Developmental Biology, University of Colorado at Boulder, Boulder, Colorado 80309, \\ USA
}

Post-translational modifications of histones play a critical role in gene expression control. Ultimately, cancer is a disease of aberrant gene expression. Accordingly, several histone-modifying enzymes have been described as proto-oncogenes or tumor suppressors. Recent reports, including one from Shema and colleagues (pp. 26642676) in the October 1, 2008, issue of Genes and Development, indicate that deregulation of histone H2B monoubiquitination may contribute to cancer development.

Once considered mere packaging devices for DNA, nucleosomes are now regarded as key regulators of gene activity. In the past two decades, a flurry of research activity identified a myriad of post-translational modifications (PTMs) affecting the core nucleosomal histones (H2A, H2B, H3, and H4). For chromatin biologists, the challenge now resides in deciphering the biological functions of these PTMs, as well as the enzymes that regulate them. Recent advances have defined a clear role for histone $\mathrm{H} 2 \mathrm{~B}$ monoubiquitination in transcriptional regulation and, interestingly, the enzymes regulating this PTM have been linked to tumorigenesis.

H2B monoubiquitination: a dynamic modification with pleiotropic effects in transcription.

Addition of the ubiquitin peptide to a target protein is catalyzed by the coordinated action of three enzymatic activities: an ATP-dependent ubiquitin-activating enzyme (E1), an intermediate ubiquitin-conjugating enzyme (E2), and a terminal ubiquitin-protein ligase (E3). Whereas polyubiquitination marks proteins for degradation by the $26 \mathrm{~S}$ proteasome, monoubiquitination serves signaling purposes and can be reversed by the action of ubiquitin-specific proteases (USPs). In metazoans, histone $\mathrm{H} 2 \mathrm{~B}$ is monoubiquitinated at Lys-120 (corresponding to Lys-123 in Saccharomyces cerevisiae). The en-

[Keywords: RNF20; BRE1; H2B ubiquitylation; tumor suppressor; transcription]

${ }^{1}$ Correspondence.

E-MAIL Joaquin.espinosa@colorado.edu; FAX (303) $492-7744$.

Article is online at http://www.genesdev.org/cgi/doi/10.1101/gad.1732108. zymes catalyzing $\mathrm{H} 2 \mathrm{~B}$ monoubiquitination (ubH2B) were first identified in S. cerevisiae: Rad6 (E2) and Bre1 (E3) (see Table 1 for a list of enzymes with known roles in H2B ubiquitination; Robzyk et al. 2000; Hwang et al. 2003; Wood et al. 2003). In humans, there are two homologs of Rad6 (HR6A and HR6B) and Bre1 (RNF20 and RNF40) (Koken et al. 1991; Kim et al. 2005). HR6B knockout mice are viable, display male sterility, and have normal levels of ubH2B, which suggests that HR6A and HR6B may play redundant roles in H2B monoubiquitination, or that there are other ubH2B E2s in mammalian cells (Roest et al. 1996). In this regard, the E2 UbcH6 has been shown to cooperate with RNF20/ RNF40 to mediate H2B monoubiquitination in vitro, yet its contribution to ubH2B in vivo remains to be defined (Zhu et al. 2005). RNF20 and RNF40 form a complex in vivo, and knockdown of RNF20 (but not of RNF40) significantly reduces levels of ubH2B in human cells (Kim et al. 2005; Zhu et al. 2005). In S. cerevisiae, H2B monoubiquitination can be reversed by two USPs: Ubp8 and Ubp10. Ubp8 is a component of the transcriptional coactivator complex SAGA (Spt-Ada-Gcn5-acetyltransferase) and deubiquitinates ubH2B both in vivo and in vitro (Henry et al. 2003; Daniel et al. 2004). In humans, the Ubp8 ortholog USP22 is part of the coactivator acetyltransferase complex hSAGA/TFTC/STAGA (Zhang et al. 2008; Zhao et al. 2008). In contrast, Ubp10 (also known as Dot4) acts independently of SAGA, and interacts instead with the protein Sir4 to facilitate silencing of subtelomeric and rDNA chromatin in S. cerevisiae (Emre et al. 2005; Gardner et al. 2005). A role for H2B deubiquitination in gene silencing has also been proposed in flies, where the ubH2B deubiquitinase USP7 has been shown to mediated epigenetic silencing of homeotic genes (van der Knaap et al. 2005).

$\mathrm{ubH} 2 \mathrm{~B}$ has been clearly implicated in transcriptional regulation, with both positive and negative overall effects on gene activity being apparent. Several lines of evidence support a positive role for $\mathrm{ubH} 2 \mathrm{~B}$ in transcription: (1) Bre1/RNF20 is recruited to promoters by DNAbinding transactivators such as Gal4 in yeast and p53 in humans. $\mathrm{ubH} 2 \mathrm{~B}$ is required for full induction of certain Gal4 target genes (Henry et al. 2003; Hwang et al. 2003). Concurrent overexpression of RNF20 and p53 enhances induction of the p53 target genes p21 and MDM2, and 
Table 1. Human enzymes linked to H2B ubiquitination

\begin{tabular}{lcclc}
\hline Human protein & S. cerevisiae counterpart & Activity in human cells & Known substrates & Role in cancer \\
\hline RNF20 & Bre1 & E3 ligase & H2B & Tumor suppressor \\
RNF40 & Bre1 & E3 ligase? & & \\
HR6A/B & Rad6 & E2 ligase & H2B & Oncogene \\
UbcH6 & & E2 ligase & H2B, H2A & Oncogene \\
USP22 & Ubp8 & Deubiquitinase & MDM2, H2B, p53 \\
USP7/HAUSP & & Deubiquitinase & p53, H2A? H2B? & Oncogen \\
MDM2 & & E3 ligase & &
\end{tabular}

knockdown of RNF20 reduces activity of both promoters in reporter assays (Kim et al. 2005). (2) ubH2B triggers a "trans-histone" modification cascade, as trimethylation of Lys-4 and Lys-79 in histone H3 requires ubH2B (Briggs et al. 2002; Sun and Allis 2002; Kim et al. 2005). These methylation marks are positively associated with gene activity. In yeast, this "histone cross-talk" is mediated by Csp35, a protein that interacts with both the H3-K4 methyl-transferase complex COMPASS and the H3-K79 methyltransferase Dot1. Importantly, Csp35 recruitment to promoters requires ubH2B (Lee et al. 2007). (3) Chromatin immunoprecipitation plus microarray analysis (ChIP-on-chip) of ubH2B in human cells revealed that this modification associates preferentially with the intragenic region of highly expressed genes (Minsky et al. 2008). Accordingly, Rad6 and Brel associate with the transcription elongation complex in yeast cells (Xiao et al. 2005). Furthermore, Rad6/Bre1-mediated ubH2B is abolished by mutations in subunits of protein complexes involved in transcriptional elongation and/or cotranscriptional RNA processing, such as kin28 (the kinase subunit of TFIIH, CDK7 in humans), Bur1/Bur2 cyclindependent kinases (yeast homologs of human CDK9, the kinase subunit of Positive Transcription Elongation Factor B, PTEF-b), as well as subunits of the yeast PAF complex, which associates with elongating RNA polymerase II (RNAPII) and plays diverse roles in elongation and RNA processing (for a recent review, see Weake and Workman 2008). (4) ubH2B is a positive regulator of FACT. FACT (Facilitates Chromatin Transcription) is a nucleosome remodeling/histone chaperone complex formed by SSRP1 and Spt16 that displays dual activities in transcription elongation, as it mediates both nucleosome eviction in front of the elongation complex and nucleosome reassembly after passage of the polymerase (Orphanides et al. 1999; Schwabish and Struhl 2004). In vitro transcription studies demonstrated that ubH2B cooperates with FACT to promote transcriptional elongation through nucleosome arrays (Pavri et al. 2006). More recently, in vivo experiments in yeast cells showed that $\mathrm{ubH} 2 \mathrm{~B}$ is also likely required for the histone deposition activity of FACT, as H2B-K123 mutants display diminished nucleosome reassembly in the wake of elongating polymerase (Fleming et al. 2008). Importantly, ubH2B is required for full recruitment of Spt16 to intragenic regions and the coordinated action of ubH2B and FACT in chromatin reassembly during elongation seems to prevent the inappropriate firing of intragenic cryptic pro- moters (Fleming et al. 2008). (5) As mentioned above, two USPs capable of deubiquitinating ubH2B are associated with gene silencing (Ubp10 in yeast and USP7 in flies). Mutations in these USPs leads to derepression of genes within otherwise silent chromatin domains (Emre et al. 2005; Gardner et al. 2005; van der Knaap et al. 2005). In summary, ubH2B seems to promote gene activity by multiple means, including positive effects on other histone modifications associated with active transcription, as well as RNAPII elongation.

However, a uniform view of ubH2B in transcriptional control is prevented by findings suggesting that $\mathrm{ubH} 2 \mathrm{~B}$ represses gene activity in some scenarios: (1) Ubp8, one of two ubH2B deubiquitinases found in yeast, is a subunit of the coactivator complex SAGA and is required for SAGA-dependent gene activation (Henry et al. 2003). Interestingly, Ubp8 promotes RNAPII phosphorylation in Ser2 of its C-terminal domain (CTD) repeats (Wyce et al. 2007). Ser2-phosphorylation is a mark of transcription elongation and is required for cotranscriptional recruitment of mRNA processing factors (Ahn et al. 2004). Ubp8 is required for recruitment of the Ser2-kinase Ctk1, but this requirement is lost in Brel mutants, indicating that ubH2B somehow prevents Ctk1 recruitment and Ser2 phosphorylation. Like Rad6 and Bre1, Ubp8 also associates with intragenic regions, suggesting that multiple rounds of $\mathrm{H} 2 \mathrm{~B}$ ubiquitination and deubiquitination are required for full gene induction (Henry et al. 2003; Wyce et al. 2007). Alternatively, we could envision a "checkpoint" model, where negative effects of ubH2B in early elongation may allow for RNAPII pausing and coordination of early elongation with RNA processing. (2) In human cells, USP22 (the H2B deubiquitinase in hSAGA/TFTC/STAGA) is required for full activation of several genes, including some in the transcriptional networks governed by p53, MYC, and androgen receptor (AR) (Zhang et al. 2008; Zhao et al. 2008). These seemingly contradictory observations on the role of ubH2B on transcription can be reconciled if we accept that a dynamic control of $\mathrm{ubH} 2 \mathrm{~B}$ is required to fine-tune gene activity. Unlike the straightforward action of histone acetyltransferases and deacetylases that seem to control transcription through an on-off switch, the ultimate effect of ubH2B on RNAPII activity may differ from gene to gene, or among different chromatin landscapes (as defined by the presence of other histone PTMs or the local nucleosome architecture) or even perhaps between the proximal promoter region versus the intragenic re- 
gion of a given transcription unit. In this regard, a recent study by Shema et al. (2008) began to illuminate the context-dependent functions of ubH2B via analysis of RNF20 in human cells.

\section{hBRE1/RNF20: a gene-specific regulator that suppresses tumor growth?}

In order to investigate the role of RNF20 in transcriptional regulation at a genome-wide scale, Shema et al. (2008) performed a microarray analysis of gene expression in Hela cells upon RNF20 knockdown. Despite the fact that RNF20 depletion produced a significant reduction in the cellular pool of ubH2B, only a modest fraction of the total transcriptome was affected and cell viability was not compromised. RNF20 depletion led to both down-regulation and up-regulation of distinct subsets of genes. Using ChIP-seq, ChIP-on-chip, and published data on genome-wide chromatin architecture, Shema et al. (2008) arrived at several important conclusions: (1) RNF20-suppressed genes display significantly higher levels of ubH2B as compared with RNF20-dependent and RNF20-independent genes, supporting the notion that RNF20 can repress transcription by increasing ubH2B levels at specific loci. Despite the differences on overall ubH2B levels, no significant differences in ubH2B localization were apparent among groups, as all three subsets showed (on average) a peak of ubH2B occupancy within the first kilobase downstream from the transcription start site, with the signal dropping steadily toward the $3^{\prime}$ end of genes. Thus, the repressive effects of RNF20 and ubH2B would take place during early elongation. (2) RNF20-suppressed genes are (on average) expressed at much lower levels than RNF20-dependent genes. However, RNF20-suppressed genes carry significantly higher levels of RNAPII, trimethylated H3-K4, and acetylated $\mathrm{H} 3(\mathrm{~K} 8, \mathrm{~K} 14)$ at the $5^{\prime}$ end of the transcription units, yet they exist within "closed" chromatin domains. Again, these results support the notion that RNF20-mediated H2B ubiquitination represses transcription at postRNAPII recruitment steps and leads to polymerase pausing during early elongation. Once considered a rare phenomenon, RNAPII preloading and pausing is now known to be a widespread regulatory feature among many inducible genes activated by developmental cues, stress, or rapid intracellular signaling (Margaritis and Holstege 2008). In contrast, housekeeping genes constitutively expressed at high levels show fewer signs of RNAPII pausing. Interestingly, Shema et al. (2008) found that many serum-responsive genes are suppressed by RNF20. Genes in this category, like the proto-oncogenes, MYC and FOS, were among the first where RNAPII pausing was characterized (Krumm et al. 1992; Glover-Cutter et al. 2008). Depletion of RNF20 leads to increased basal and induced levels of these and many other EGF-responsive genes, such as RHOB, NR4A2, ITGA6, FOSL2, and TM4SF1, whereas housekeeping genes like GAPDH and NADH dehydrogenase were not affected. (3) RNF20 repressive effects on transcription are largely independent of effects on H3K4 trimethylation. Unlike that observed in HEK-293T cells, where RNF20 knockdown recapitulated the "trans-histone" pathway first observed in yeast (Kim et al. 2005), Shema et al. (2008) did not observe significant changes in global levels of $\mathrm{H} 3-\mathrm{K} 4 \mathrm{me} 3$ in Hela cells depleted for RNF20. Furthermore, ChIP-onchip analysis demonstrated that most (but not all) RNF20-regulated genes do not display changes in H3K4me3, despite the loss of ubH2B. These results question the prevalence of the "trans-histone pathway" (ubH2B $\Rightarrow$ H3K4me3/H3K79me3), which may not occur in all human cell types or it may take place on specific subsets of genes.

The fact that RNF20 behaved as a suppressor of EGFsignaling prompted Shema et al. (2008) to investigate a possible role for RNF20 in oncogenic cell behaviors, such as cell migration, growth in soft agar, and the ability of cells to form tumors in xenograft models. Interestingly, RNF20-depleted cells showed higher oncogenic potential in all of these assays, suggesting that RNF20 may function as a tumor suppressor. This notion was further supported by the discovery that RNF20 depletion leads to $>10$-fold decrease in expression of the tumor suppressor p53! In fact, RNF20-depleted cells displayed attenuated p53-dependent apoptosis and cell cycle arrest in diverse cell types. Accordingly, RNF20 knockdown led to reduced expression of many canonical p53 target genes such as GDF15/MIC1, C12orf5/TIGAR, SERPINB5/ Maspin, BAX, FDXR, CDKN1A/p21, and TP53I3/PIG3. If we add to this the fact that RNF20 had been previously characterized as a p53-binding protein capable of stimulating p53-dependent transcription (Kim et al. 2005), we could safely conclude at this point that RNF20 plays positive roles within this tumor suppressor transcriptional network. Finally, Shema et al. (2008) found that RNF20 is commonly silenced via DNA hypermethylation in breast cancers, suggesting that tumor cells shut down RNF20 during cancer progression. Taken together, the study by Shema et al. (2008) advances significantly our understanding of the context-dependent functions of $\mathrm{ubH} 2 \mathrm{~B}$ in transcriptional regulation and proposes a role for ubH2B deregulation in cancer development.

\section{USP22: a gene-specific regulator that promotes tumor growth?}

If we accept that hBRE1/RNF20 is indeed a tumor suppressor, should we then propose that USP22, the subunit of hSAGA/TFTC/STAGA that catalyzes the opposite enzymatic reaction, is an oncoprotein? Recent advances in transcriptional profiling of tumor cells generated an 11gene signature associated with poor prognosis (i.e., short interval to disease recurrence, distant metastasis and death after therapy) (Glinsky et al. 2005). This gene signature can predict treatment outcome with unprecedented prowess across diverse cancer types, suggesting the presence of a conserved pathway driving malignancy in a wide range of organs. Increased expression of seven genes in the signature is strongly correlated with malignancy: Ki67, CCNB1, GBX2, BUB1, KNTC2, BMI1, RNF2, and last (but not least) . . USP22!! Ki67, a protein 
recognized by mAb ki-67, is a marker of cell proliferation. CCNB1 is cyclin B1, the cyclin partner for CDC2 and a key driver of mitosis. GBX2 (gastrulation homeobox 2) is a transcriptional regulator known to stimulate growth of human prostate cancer cells. BUB1 and KTNC2 are part of the spindle assembly mitotic checkpoint and it is hypothesized that their overexpression may lead to aberrant checkpoint function and consequent aneuploidy, a hallmark of cancer. BMI1 and RNF2 are two subunits of the human Polycomb repressive complex like-1 (hPRC1L). BMI1 is potent oncogene with a well-established role in the self-renewal potential of both normal and tumor stem cells in various organs (Lessard and Sauvageau 2003). BMI1 overexpression has been observed in many leukemias as well as lung, breast, colon, and prostate carcinomas, suggesting that deregulation of Polycomb-mediated gene silencing via overexpression of BMI1 and/or RNF2 could drive malignant progression, likely via aberrant silencing of tumor suppressor genes such as INK4a/p16 and ARF/p19 (Bracken et al. 2007). Thus, the placement of USP22 within a select group of genes with known oncogenic properties supports the idea that deregulation of ubH2B may contribute to cancer.

Little is known about the biological role of USP22 in human cells, but whatever little we know may provide some hints as to how USP22 may exert oncogenic effects. Two recent reports have described a positive role for USP22 in distinct oncogenic transcriptional networks (Zhang et al. 2008; Zhao et al. 2008). Zhang et al. (2008) found that induction of MYC target genes, such as cyclin D2, is impaired in cells depleted of USP22. They showed that recruitment of USP22 to MYC target genes requires MYC activity, demonstrated that USP22 is required for MYC-driven transformation of human fibroblasts, and that USP22 knockdown produces an accumulation of cells in G1 phase of the cell cycle. These results indicate that USP22 is a positive regulator of MYC-dependent transcription and that this may account for its oncogenic properties. However, a simplified view of USP2 2 action is prevented by the observation by Zhang et al. (2008) that USP22 is also a positive regulator of p53-dependent transcription. In their report, depletion of USP22 impaired induction of the p53 target genes p21 and PIG3 upon p53 overexpression. These observations are somehow at odds with the findings by Kim et al. (2005) describing RNF20 as a coactivator of p53-dependent activation of $\mathrm{p} 21$ and MDM2. To reconcile these observations, we could hypothesize that only a correct balance between RNF20 and USP22 activities may conduce to optimal p53-dependent transcriptional activation. The issue could be clarified with a side-by-side comparison of the effects of depletion of either enzyme on endogenous p53-dependent transcription. Interestingly, Zhang et al. (2008) found that USP22 depletion had no effects on activation of the p53 target gene PUMA in their system, indicating that USP22 plays gene-specific effects within the p53 transcriptional network. Regardless of the final contribution of USP22 to p53-dependent transcription, the data provided by Zhang et al. (2008) indicates that USP22 contributes mostly to cell proliferation, likely through modulation of MYC activity. In an independent characterization of USP22, Zhao et al. (2008) reported a critical role for this protein in the AR transcriptional network. Using loss-of-function experiments both in Drosophila and human cells, they demonstrated that USP22 and two other subunits of the socalled "deubiquitinase module" of hSAGA/TFTC/ STAGA, ATXNL3, and ENY2, are required for ARmediated transactivation. AR is a potent driver of cell proliferation in the prostate epithelium, and uncontrolled AR activity contributes to the development of prostate cancer. Thus, we could speculate that overexpression of USP22 contributes to development of prostate cancer. Although not fully described in their report, Zhao et al. (2008) affirm that USP22 is also required for estrogen receptor (ER)-mediated transactivation. In this case, USP22 may also play an important role in the proliferation of the breast epithelium, and consequently, could be implicated in breast cancer.

Of note, the work by Shema et al. (2008) suggests that RNF20/ubH2B preferentially repress inducible genes carrying paused RNAPII, like those within the EGF-dependent transcriptional response. Interestingly, studies in yeast indicate that SAGA predominantly regulates expression of stress-inducible genes, rather than housekeeping genes (Huisinga and Pugh 2004). Consequently, full activation of serum-response genes may require USP22 and hSAGA/TFCT/STAGA, which could release RNAPII into elongation mode via ubH2B deubiquitination. Therefore, a testable model for the role of RNF20 and USP22/hSAGA in regulation of inducible genes repressed by ubH2B in human cells is depicted in Figure 1.

\section{USP7 is HAUSP, an ubH2B deubiquitinase mediating gene silencing in flies and a regulator of the p53-MDM2 circuit in mammals}

Despite the excitement surrounding the discovery of novel histone-modifying enzymes, we shall remember that histones may be just one, even perhaps not the most relevant, substrate for these enzymes. How many of the effects attributed to RNF20 and USP22 are truly due to regulation of $\mathrm{ubH} 2 \mathrm{~B}$, as opposed to regulation of other uncharacterized substrates? Whereas the problem is tractable in yeast, where single-copy histone genes can be replaced by mutant isoforms, it is quite insoluble in mammalian cells carrying multiple copies of these genes. One clear illustration of the pleiotropic nature of "histone-modifying enzymes" is provided by USP7/ HAUSP. As mentioned above, USP7 has been characterized as an $\mathrm{ubH} 2 \mathrm{~B}$ deubiquitinase involved in epigenetic silencing of homeotic genes in flies (van der Knaap et al. 2005). Homeotic gene silencing in fly larvae is mediated by the Polycomb silencing complex, and USP7 mutations enhance Polycomb phenotypes. Interestingly, biochemical purification of USP7 from Drosophila embryo extracts identified the metabolic enzyme GMPS (GMPsynthase) as a strong USP7 interactor required for its $\mathrm{ubH} 2 \mathrm{~B}$ deubiquitinase activity. GMPS is an enzyme re- 


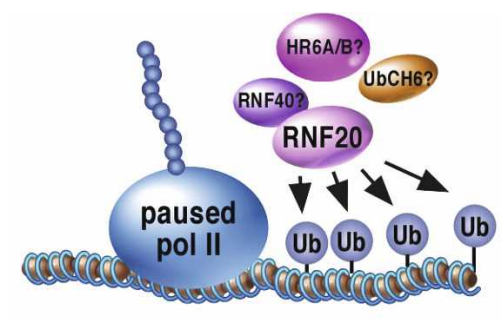

$$
\begin{gathered}
\text { activating } \\
\text { stimulus }
\end{gathered}
$$

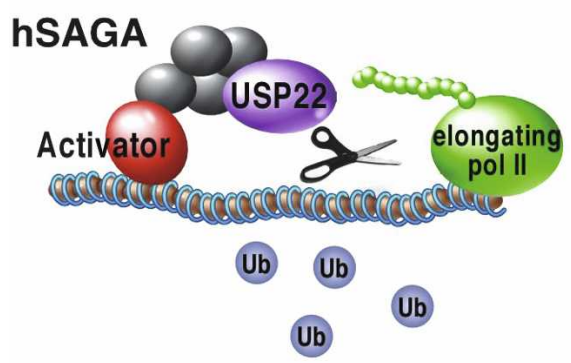

Figure 1. Model for the mechanism of action of RNF20, $\mathrm{ubH} 2 \mathrm{~B}$, and USP22 in activation of inducible genes carrying poised RNAPII. RNF20-repressed genes, such as many in the serum-response transcriptional network, carry high amounts of $\mathrm{ubH} 2 \mathrm{~B}$ and display signs of poised RNAPII, including marks associated with transcription initiation such as $\mathrm{H} 3 \mathrm{~K} 4 \mathrm{me} 3$. ubH2B occupancy is higher right downstream from transcription start sites, suggesting that it may block early elongation steps at these genes. Possible E2s for RNF20 include Ubch6 and the human homologs of Rad6. The deubiquitinase module of hSAGA, containing USP22, is required for full induction of several inducible genes, including some controlled by MYC, p53, and the AR. Thus, activators could recruit hSAGA to promoters repressed by $\mathrm{ubH} 2 \mathrm{~B}$ in order to promote elongation via $\mathrm{H} 2 \mathrm{~B}$ deubiquitination.

quired for synthesis of guanine nucleotides whose expression is strongly induced in rapidly proliferating cells (Boritzki et al. 1981). This suggests that GMPS may provide a link between the proliferative/metabolic state of the cell and histone modifications in analogous fashion to NAD-dependent histone deacetylases of the Sirtuin family. However, there is more to USP7 than histones. The human homolog of USP7, also known as HAUSP (Herpes virus-associated USP), was first identified as an interactor of the Herpes simplex virus type 1 immediateearly protein Vmw110 (Everett et al. 1998). Later, the Gu laboratory (Li et al. 2002) described HAUSP as a p53associated protein. Given that p53 stability is tightly regulated by MDM2- and ubiquitin-dependent degradation, $\mathrm{Gu}$ and colleagues (Li et al. 2002) hypothesized that HAUSP could mediate p53 deubiquitination and stabilization. Indeed, HAUSP overexpression led to p53 accumulation in their system, which prompted the conclusion that HAUSP could work as a tumor suppressor by antagonizing MDM2 function. However, elegant work by the Vogelstein laboratory (Cummins et al. 2004) reached the opposite conclusion: They showed that cells where both alleles of HAUSP had been knocked-out displayed much higher levels of p53. This led to the observation that HAUSP deubiquitinates and stabilizes not p53, but MDM2 itself! (Cummins et al. 2004; Li et al. 2004). Thus, the current model for HAUSP action indicates that in the absence of cellular stress, MDM2 is stabilized by the deubiquitinase activity of HAUSP, which results in low levels of p53. Upon cellular stress, the MDM2-HAUSP interaction is blocked likely via PTMs, MDM2 is destabilized by autoubiquitination, and free HAUSP molecules could eventually collaborate in stress-induced stabilization of p53. The list of HAUSP targets has been recently increased with the addition of the FOXO4 transcription factor, which requires monoubiquitination for full transactivation potential and is therefore deactivated by HAUSP (van der Horst et al. 2006). What is the contribution of different USP7/ HAUSP targets to its overall cellular function? Is HAUSP an ubH2B deubiquitinase in human cells? If so, can HAUSP be targeted to p53 target genes to modulate p53-dependent transcription? All in all, these studies on USP7/HAUSP remind us to be cautious in our interpretation of the effects produced by depletion of RNF20, USP22, and other deubiquitinases in human cells, as some of these effects may be independent of ubH2B.

\section{MDM2 and BRCA1: novel histone E3 ligases?}

Another possible connection between ubH2B and malignant transformation is provided by reports describing MDM2, a potent oncogene, and BRCA1, a tumor suppressor, as H2B E3 ligases. Previous work by the Oren group (Minsky and Oren 2004) reported that MDM2 can ubiquitinate both $\mathrm{H} 2 \mathrm{~A}$ and $\mathrm{H} 2 \mathrm{~B}$ and proposed that this activity would lead to repression of p53 target genes. Importantly, MDM2 is unlikely to contribute to ubiquitination of H2B-K120 in vivo, as mutation of K120 did not affect MDM2-mediated H2B ubiquitination. Instead, K125 in H2B seemed to be the target lysine for MDM2. Nothing is known about the role of H2B K125 ubiquitination and additional work will be required to elucidate the impact of this PTM in gene expression. Interestingly, MDM2 seemed to ubiquitinate H2A more potently than H2B. In humans, H2A monoubiquitination has clear roles in gene silencing and is catalyzed by two E3 ligases, 2A-HUB and Ring1B, which associate with transcriptional repressor complexes (for review, see Weake and Workman 2008). Thus, it is possible that MDM2 may mediate silencing of p53-target genes via $\mathrm{H} 2 \mathrm{~A}$, rather than H2B monoubiquitination. BRCA1, a RING fingercontaining protein, can also catalyze ubiquitination of both $\mathrm{H} 2 \mathrm{~A}$ and $\mathrm{H} 2 \mathrm{~B}$ in vitro, yet the in vivo relevance of these activity remains to be defined (Mallery et al. 2002; $\mathrm{Xia}$ et al. 2003). The E3 ligase activity of BRCA1 is stimulated by the interacting protein BARD1 (Xia et al. 2003). It is accepted that BRCA1 and BARD1 cooperate in tumor suppression, likely through their roles in repair of double-stranded breaks. Of note, Rad6, the main E2 for ubH2B in yeast, is also involved in DNA damage repair, 
although targets other than $\mathrm{H} 2 \mathrm{~B}$ may be relevant in this context (for review, see Osley and Shen 2006). Additional work will be required to dissect the contribution of BRCA1/BARD1-mediated H2B ubiquitination to their overall tumor suppressive activity.

\section{Final remarks}

As our understanding of histone PTMs, the enzymes that catalyze them, and their role in cancer development increases, so does our capacity to envision therapeutic strategies aimed at manipulating the transcriptome of cancer cells for their selective elimination. Recent successes in the development of specific small-molecule inhibitors of the E3 ligase activity of MDM2 and the MDM2-p53 interaction (Vassilev et al. 2004; Yang et al. 2005) indicate that similar strategies could be used for other E2 and E3 ligases, including those modulating H2B ubiquitination.

\section{Acknowledgments}

I thank my graduate students Aaron Donner and Nathan Gomes for careful review of the manuscript, and Dr. Ali Shilatifard for discussions on ubH2B. Work in my laboratory is supported by grants from NIH (CA117907) and the Cancer League of Colorado.

\section{References}

Ahn, S.H., Kim, M., and Buratowski, S. 2004. Phosphorylation of serine 2 within the RNA polymerase II C-terminal domain couples transcription and $3^{\prime}$ end processing. Mol. Cell 13: 67-76.

Boritzki, T.J., Jackson, R.C., Morris, H.P., and Weber, G. 1981. Guanosine-5'-phosphate synthetase and guanosine-5'-phosphate kinase in rat hepatomas and kidney tumors. Biochim. Biophys. Acta 658: 102-110.

Bracken, A.P., Kleine-Kohlbrecher, D., Dietrich, N., Pasini, D., Gargiulo, G., Beekman, C., Theilgaard-Monch, K., Minucci, S., Porse, B.T., Marine, J.C., et al. 2007. The Polycomb group proteins bind throughout the INK4A-ARF locus and are disassociated in senescent cells. Genes \& Dev. 21: 525-530.

Briggs, S.D., Xiao, T., Sun, Z.W., Caldwell, J.A., Shabanowitz, J., Hunt, D.F., Allis, C.D., and Strahl, B.D. 2002. Gene silencing: Trans-histone regulatory pathway in chromatin. Nature 418: 498.

Cummins, J.M., Rago, C., Kohli, M., Kinzler, K.W., Lengauer, C., and Vogelstein, B. 2004. Tumour suppression: Disruption of HAUSP gene stabilizes p53. Nature doi: 10.1028/ nature02501.

Daniel, J.A., Torok, M.S., Sun, Z.W., Schieltz, D., Allis, C.D., Yates 3rd, J.R., and Grant, P.A. 2004. Deubiquitination of histone H2B by a yeast acetyltransferase complex regulates transcription. J. Biol. Chem. 279: 1867-1871.

Emre, N.C., Ingvarsdottir, K., Wyce, A., Wood, A., Krogan, N.J., Henry, K.W., Li, K., Marmorstein, R., Greenblatt, J.F., Shilatifard, A., et al. 2005. Maintenance of low histone ubiquitylation by Ubp10 correlates with telomere-proximal Sir2 association and gene silencing. Mol. Cell 17: 585-594.

Everett, R.D., Freemont, P., Saitoh, H., Dasso, M., Orr, A., Kathoria, M., and Parkinson, J. 1998. The disruption of ND10 during herpes simplex virus infection correlates with the Vmw110- and proteasome-dependent loss of several PML isoforms. J. Virol. 72: 6581-6591.

Fleming, A.B., Kao, C.F., Hillyer, C., Pikaart, M., and Osley, M.A. 2008. H2B ubiquitylation plays a role in nucleosome dynamics during transcription elongation. Mol. Cell 31: 5766.

Gardner, R.G., Nelson, Z.W., and Gottschling, D.E. 2005 Ubp10/Dot4p regulates the persistence of ubiquitinated histone H2B: Distinct roles in telomeric silencing and general chromatin. Mol. Cell. Biol. 25: 6123-6139.

Glinsky, G.V., Berezovska, O., and Glinskii, A.B. 2005. Microarray analysis identifies a death-from-cancer signature predicting therapy failure in patients with multiple types of cancer. J. Clin. Invest. 115: 1503-1521.

Glover-Cutter, K., Kim, S., Espinosa, J., and Bentley, D.L. 2008 RNA polymerase II pauses and associates with pre-mRNA processing factors at both ends of genes. Nat. Struct. Mol. Biol. 15: 71-78.

Henry, K.W., Wyce, A., Lo, W.S., Duggan, L.J., Emre, N.C., Kao, C.F., Pillus, L., Shilatifard, A., Osley, M.A., and Berger, S.L. 2003. Transcriptional activation via sequential histone $\mathrm{H} 2 \mathrm{~B}$ ubiquitylation and deubiquitylation, mediated by SAGA-associated Ubp8. Genes \& Dev. 17: 2648-2663.

Huisinga, K.L. and Pugh, B.F. 2004. A genome-wide housekeeping role for TFIID and a highly regulated stress-related role for SAGA in Saccharomyces cerevisiae. Mol. Cell 13: 573 585.

Hwang, W.W., Venkatasubrahmanyam, S., Ianculescu, A.G., Tong, A., Boone, C., and Madhani, H.D. 2003. A conserved RING finger protein required for histone H2B monoubiquitination and cell size control. Mol. Cell 11: 261-266.

Kim, J., Hake, S.B., and Roeder, R.G. 2005. The human homolog of yeast BRE1 functions as a transcriptional coactivator through direct activator interactions. Mol. Cell 20: 759-770.

Koken, M.H., Reynolds, P., Jaspers-Dekker, I., Prakash, L., Prakash, S., Bootsma, D., and Hoeijmakers, J.H. 1991. Structural and functional conservation of two human homologs of the yeast DNA repair gene RAD6. Proc. Natl. Acad. Sci. 88: 8865-8869.

Krumm, A., Meulia, T., Brunvand, M., and Groudine, M. 1992. The block to transcriptional elongation within the human c-myc gene is determined in the promoter-proximal region. Genes \& Dev. 6: 2201-2213.

Lee, J.S., Shukla, A., Schneider, J., Swanson, S.K., Washburn, M.P., Florens, L., Bhaumik, S.R., and Shilatifard, A. 2007. Histone crosstalk between $\mathrm{H} 2 \mathrm{~B}$ monoubiquitination and $\mathrm{H} 3$ methylation mediated by COMPASS. Cell 131: 1084-1096.

Lessard, J. and Sauvageau, G. 2003. Bmi-1 determines the proliferative capacity of normal and leukaemic stem cells. $\mathrm{Na}$ ture 423: 255-260.

Li, M., Chen, D., Shiloh, A., Luo, J., Nikolaev, A.Y., Qin, J., and $\mathrm{Gu}, \mathrm{W} .2002$. Deubiquitination of p53 by HAUSP is an important pathway for p53 stabilization. Nature 416: 648-653.

Li, M., Brooks, C.L., Kon, N., and Gu, W. 2004. A dynamic role of HAUSP in the p53-Mdm2 pathway. Mol. Cell 13: 879886.

Mallery, D.L., Vandenberg, C.J., and Hiom, K. 2002. Activation of the E3 ligase function of the BRCA1/BARD1 complex by polyubiquitin chains. EMBO J. 21: 6755-6762.

Margaritis, T. and Holstege, F.C. 2008. Poised RNA polymerase II gives pause for thought. Cell 133: 581-584.

Minsky, N. and Oren, M. 2004. The RING domain of Mdm2 mediates histone ubiquitylation and transcriptional repression. Mol. Cell 16: 631-639.

Minsky, N., Shema, E., Field, Y., Schuster, M., Segal, E., and 
Oren, M. 2008. Monoubiquitinated H2B is associated with the transcribed region of highly expressed genes in human cells. Nat. Cell Biol. 10: 483-488.

Orphanides, G., Wu, W.H., Lane, W.S., Hampsey, M., and Reinberg, D. 1999. The chromatin-specific transcription elongation factor FACT comprises human SPT16 and SSRP1 proteins. Nature 400: 284-288.

Osley, M.A. and Shen, X. 2006. Altering nucleosomes during DNA double-strand break repair in yeast. Trends Genet. 22: 671-677.

Pavri, R., Zhu, B., Li, G., Trojer, P., Mandal, S., Shilatifard, A., and Reinberg, D. 2006. Histone H2B monoubiquitination functions cooperatively with FACT to regulate elongation by RNA polymerase II. Cell 125: 703-717.

Robzyk, K., Recht, J., and Osley, M.A. 2000. Rad6-dependent ubiquitination of histone H2B in yeast. Science 287: 501504.

Roest, H.P., van Klaveren, J., de Wit, J., van Gurp, C.G., Koken, M.H., Vermey, M., van Roijen, J.H., Hoogerbrugge, J.W., Vreeburg, J.T., Baarends, W.M., et al. 1996. Inactivation of the HR6B ubiquitin-conjugating DNA repair enzyme in mice causes male sterility associated with chromatin modification. Cell 86: 799-810.

Schwabish, M.A. and Struhl, K. 2004. Evidence for eviction and rapid deposition of histones upon transcriptional elongation by RNA polymerase II. Mol. Cell. Biol. 24: 10111-10117.

Shema, E., Tirosh, I., Aylon, Y., Huang, J., Ye, C., Moskovits, N., Raver-Shapira, N., Minsky, N., Pirngruber, J., Tarcic, G., et al. 2008. The histone H2B-specific ubiquitin ligase RNF20/ hBRE1 acts as a putative tumor suppressor through selective regulation of gene expression. Genes \& Dev. 22: 2664-2676.

Sun, Z.W. and Allis, C.D. 2002. Ubiquitination of histone H2B regulates $\mathrm{H} 3$ methylation and gene silencing in yeast. $\mathrm{Na}$ ture 418: 104-108.

van der Horst, A., de Vries-Smits, A.M., Brenkman, A.B., van Triest, M.H., van den Broek, N., Colland, F., Maurice, M.M., and Burgering, B.M. 2006. FOXO4 transcriptional activity is regulated by monoubiquitination and USP7/HAUSP. Nat. Cell Biol. 8: 1064-1073.

van der Knaap, J.A., Kumar, B.R., Moshkin, Y.M., Langenberg, K., Krijgsveld, J., Heck, A.J., Karch, F., and Verrijzer, C.P. 2005. GMP synthetase stimulates histone H2B deubiquitylation by the epigenetic silencer USP7. Mol. Cell 17: 695-707.

Vassilev, L.T., Vu, B.T., Graves, B., Carvajal, D., Podlaski, F., Filipovic, Z., Kong, N., Kammlott, U., Lukacs, C., Klein, C., et al. 2004. In vivo activation of the p53 pathway by smallmolecule antagonists of MDM2. Science 303: 844-848.

Weake, V.M. and Workman, J.L. 2008. Histone ubiquitination: Triggering gene activity. Mol. Cell 29: 653-663.

Wood, A., Krogan, N.J., Dover, J., Schneider, J., Heidt, J., Boateng, M.A., Dean, K., Golshani, A., Zhang, Y., Greenblatt, J.F., et al. 2003. Bre1, an E3 ubiquitin ligase required for recruitment and substrate selection of Rad6 at a promoter. Mol. Cell 11: 267-274.

Wyce, A., Xiao, T., Whelan, K.A., Kosman, C., Walter, W., Eick, D., Hughes, T.R., Krogan, N.J., Strahl, B.D., and Berger, S.L. 2007. H2B ubiquitylation acts as a barrier to Ctk1 nucleosomal recruitment prior to removal by Ubp8 within a SAGArelated complex. Mol. Cell 27: 275-288.

Xia, Y., Pao, G.M., Chen, H.W., Verma, I.M., and Hunter, T. 2003. Enhancement of BRCA1 E3 ubiquitin ligase activity through direct interaction with the BARD1 protein. J. Biol. Chem. 278: 5255-5263.

Xiao, T., Kao, C.F., Krogan, N.J., Sun, Z.W., Greenblatt, J.F., Osley, M.A., and Strahl, B.D. 2005. Histone H2B ubiquitylation is associated with elongating RNA polymerase II. Mol.
Cell. Biol. 25: 637-651.

Yang, Y., Ludwig, R.L., Jensen, J.P., Pierre, S.A., Medaglia, M.V., Davydov, I.V., Safiran, Y.J., Oberoi, P., Kenten, J.H., Phillips, A.C., et al. 2005. Small molecule inhibitors of HDM2 ubiquitin ligase activity stabilize and activate p53 in cells. Cancer Cell 7: 547-559.

Zhang, X.Y., Varthi, M., Sykes, S.M., Phillips, C., Warzecha, C., Zhu, W., Wyce, A., Thorne, A.W., Berger, S.L., and McMahon, S.B. 2008. The putative cancer stem cell marker USP22 is a subunit of the human SAGA complex required for activated transcription and cell-cycle progression. Mol. Cell 29: 102-111.

Zhao, Y., Lang, G., Ito, S., Bonnet, J., Metzger, E., Sawatsubashi, S., Suzuki, E., Le Guezennec, X., Stunnenberg, H.G., Krasnov, A., et al. 2008. A TFTC/STAGA module mediates histone $\mathrm{H} 2 \mathrm{~A}$ and $\mathrm{H} 2 \mathrm{~B}$ deubiquitination, coactivates nuclear receptors, and counteracts heterochromatin silencing. Mol. Cell 29: 92-101.

Zhu, B., Zheng, Y., Pham, A.D., Mandal, S.S., Erdjument-Bromage, H., Tempst, P., and Reinberg, D. 2005. Monoubiquitination of human histone $\mathrm{H} 2 \mathrm{~B}$ : The factors involved and their roles in HOX gene regulation. Mol. Cell 20: 601-611. 


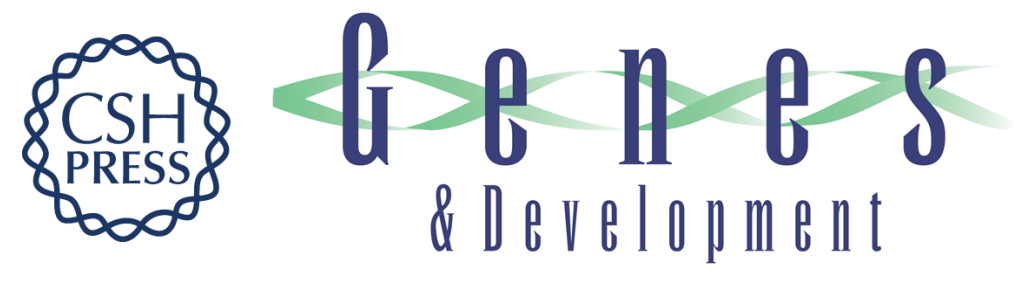

\section{Histone H2B ubiquitination: the cancer connection}

Joaquín M. Espinosa

Genes Dev. 2008, 22:

Access the most recent version at doi:10.1101/gad.1732108

Related Content The histone H2B-specific ubiquitin ligase RNF20/hBRE1 acts as a putative tumor suppressor through selective regulation of gene expression Efrat Shema, Itay Tirosh, Yael Aylon, et al.

Genes Dev. October, 2008 22: 2664-2676

References This article cites 45 articles, 14 of which can be accessed free at:

http://genesdev.cshlp.org/content/22/20/2743.full.html\#ref-list-1

Articles cited in:

http://genesdev.cshlp.org/content/22/20/2743.full.html\#related-urls

\section{License}

Email Alerting Receive free email alerts when new articles cite this article - sign up in the box at the top

Service right corner of the article or click here.

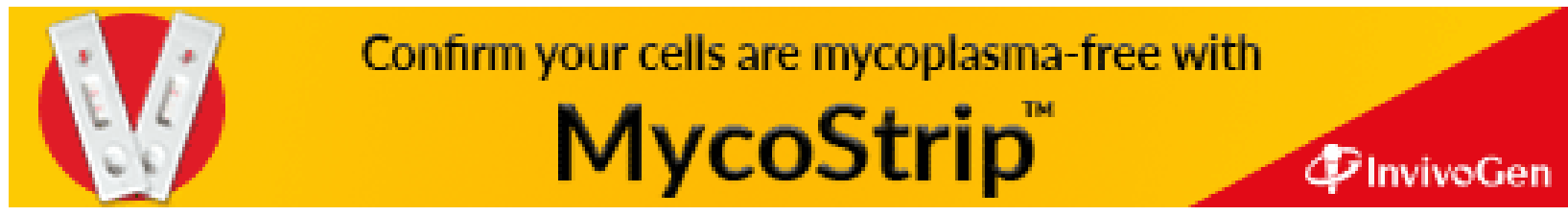

